(C) 1996 IEEE. Personal use of this material is permitted. However, permission to reprint/republish this material

for advertising or promotional purposes or for creating new collective works for resale or redistribution to servers

or lists, or to reuse any copyrighted component of this work in other works must be obtained from the IEEE.

\title{
SPATIAL-TEMPORAL HYSTERESIS EFFECTS IN AN INTENSE ELECTRON BEAM
}

\author{
A. V. Agafonov, A. N. Lebedev, V. S. Voronin \\ P. N. Lebedev Physical Institute, Leninsky Prosp. 53, Moscow 117924, Russia
}

\begin{abstract}
An electron gun with large compression of a thin annular beam for generation of high-power microwaves and IR radiation has been designed. Computer simulations of beam formation in the gun show some hysteresis effects: a beam with different current can exist in the same external conditions. At the upper (metastable) part of a hysteresis curve the beam current exceeds Child-Langmuir current. Experimental observation of these metastable states is possible if one has the possibility for fast control of emitted current, which is the case for photoemission cathode. The results of computer simulation are presented and physics reasons of such behaviour are discussed.
\end{abstract}

\section{INTRODUCTION}

In sources of electron beams of high brightness, applicable and developed for FELs and linear colliders, there have begun to be used in recent times photocathodes that permit forming "cold" beams initially. One of the important problems in these investigations concerns the magnitude of current taken from the photocathode. In a number of works - theoretical as well as experimental - there is discussed the possibility of obtaining currents exceeding the Child-Langmuir limit and is indicated that such an increase is possible only for the generation of short bunches, the length of which are less or of the order of the gap length.

Naturally, the classical CL-law is applicable only for a stationary regime with current limited by space charge for the following conditions: 1) voltage constant, 2) transverse dimensions of the emitting surface significantly exceeding (geometry close to unidimensional) the characteristic longitudinal dimensions of the accelerating region and 3) emissive power of the cathode unlimited.

Let us consider two first of the limitations. The first corresponds to constancy of current and implicitly implies that the variable quantity can be only voltage, while the value of current is determined by this voltage. It should be noted that with the appearance of efficient photoemitters and lasers, it became possible to independently vary emission current, in addition to the possibility of varying voltage. The possibility of controlling beam current in photoemission sources at fixed voltage leads to a qualitatively different situation. To a certain extent it is suggestive of the process of beam injection (although at very low initial energy) in an accelerating region rather than drawing the beam from an emitting surface.

The second limitation reduced the problem to unidimensional and, evidently, is violated under real conditions. Although the influence of the end effects is recognized, its contribution is considered to be small. At the same time, in a plane diode, when the transverse dimensions of the emitter are approximately equal to the length of the gap, the influence of fringe effects becomes determining. They are no longer simply fringe effects, they are main effects.

In view of the non-stationary nature and multi-dimensionality of the problem, the results given below were obtained by computer simulation with electromagnetic code KARAT [1].

\section{DRIFT REGION. FRINGER OR MAIN?}

For a purely stationary flow that is unidimensional and unlimited in the transverse direction, the dependence of the passing current $I_{\text {out }}$ on injection current $I_{i n j}$ is represented by the socalled "lambda-curve" (Fig. 1) [2].

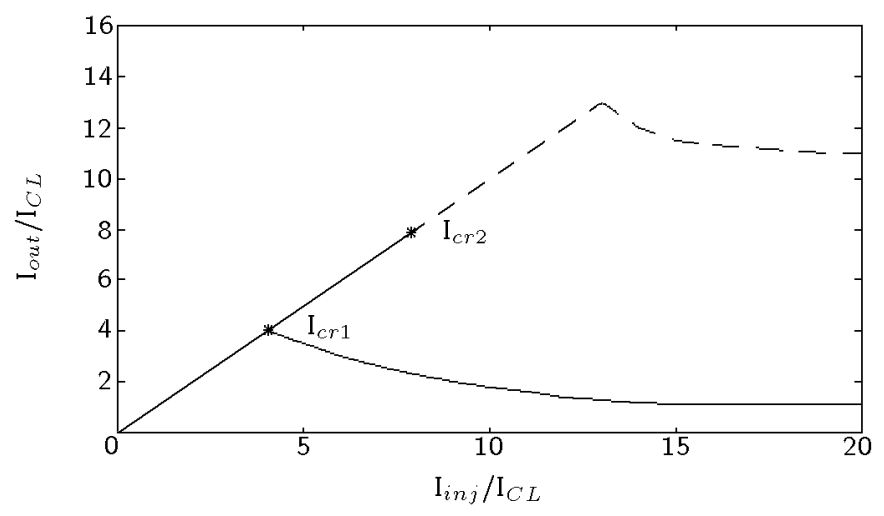

Figure. 1. "Lambda" curve

Three solutions are possible for the region from $I_{c r 1}$ to $I_{c r 2}$ in such a flow. One of these is with a virtual cathode and partial passage of the beam (lower part of solid curve) and the other two (merging into one) are without a virtual cathode and with complete passage of the beam. It is considered that the last two states are unstable and when $I_{c r 1}$ is exceeded the beam always goes to the lower part of the curve with formation of a virtual cathode. For unlimited increase of injected current the virtual cathode asymptotically approaches the plane of injection and through the region there passes only current equivalent to the CL-current for a plane diode of the same dimensions, with voltage corresponding to the energy of injected electrons. For non-relativistic energy of particles, $I_{c r 1}=4 I_{C L}, I_{c r 2}=2 I_{c r 1}$.

In [3], it was analytically shown that when the flow completely fills the chamber cross-section (the case of a seni-infinite plane slit with end plane was considered), the current passed can increase without limit $\left(I_{\text {out }} \propto \sqrt{I_{i n j}}\right)$ due to "exudation" of particles close to the chamber wall in a thin layer. The thickness of this layer decreases inversely proportionally to $\sqrt{I_{i n j}}$. Close to the end plane, the central part of the beam is blocked by the space-charge field and the virtual cathode acquires the form of such a coaxial slit with rounded edges.

Let us consider the case when two-dimensional effects appear most effective. The drift region is chosen in the form of a cylindrical resonator having the diameter $(\mathrm{D}=5 \mathrm{~cm})$ much larger than the longitudinal dimension $(\mathrm{d}=1 \mathrm{~cm})$, the diameter of injected beam $\left(2 r_{e}=1 \mathrm{~cm}\right)$ equals to the length of the resonator. We 
present the results for a beam having non-relativistic energy $\mathrm{W}$ $=20 \mathrm{keV}$. In Fig. 1, the value of current is presented in units corresponding to CL-current $\left(I_{C L}=\pi r_{e}^{2} j_{C L}, j_{C L} d^{2}=6.6 A\right.$ for 20 $\mathrm{kV}$ on the diode). The solid line show the analytical dependence for unidimensional stationary flow and the dotted lines the calculated dependence for established regime when injecting beams of various currents with a 4-ns linear front. The established beam configuration for 200-A injected current is shown in Fig.2 and is very

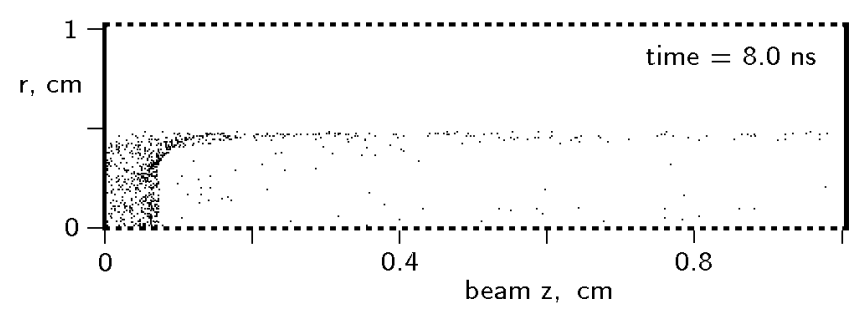

Figure. 2. Beam with "whiskers"

similar to that considered in [3]. The difference is that the beam passes far from the side walls and the passed current does not increase with the injected, but remains approximately at one level. It should be noted that the main part of the current is concentrated in "whiskers", the thickness of which decreases with increasing injected current.

The time dependence of current passing through the resonator shown in Fig. 3. The interruption of current is due to the formation of a virtual cathode arising near the center of the resonator and is rapidly displaced toward the injection plane when reflected electrons appear.

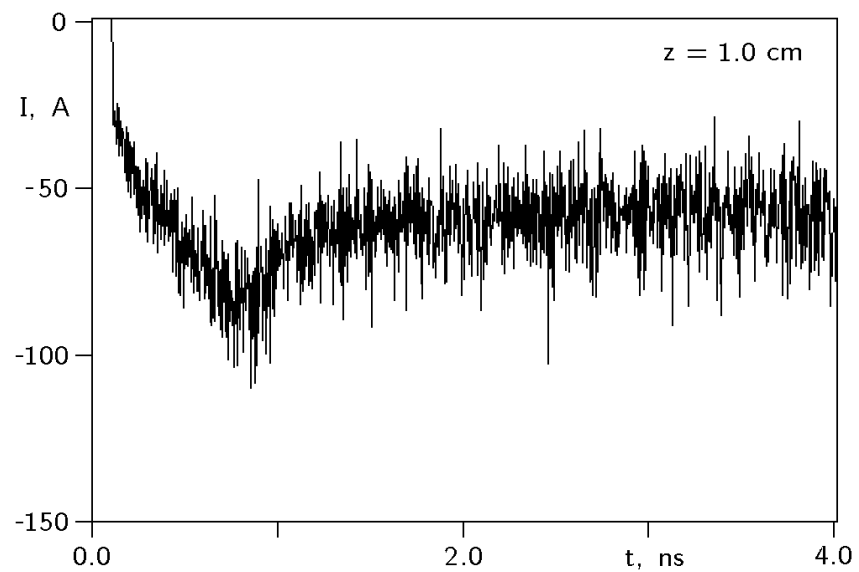

Figure. 3. Transient regime in the drift region

With increasing transverse dimensions the influence of "whiskers" on the inner part of the beam is weakened due to preventing of field by electrodes and electrons with density of current close to the Child-Langmuir begin take "emitted" from the virtual-cathode region. Since the total CL-current will increase proportionally to $r^{2}$ and the current in the "whiskers" not more than $r$, the contribution of the "whiskers" becomes small relative to current from a larger area, but lower current density. This corresponds to unidimensional flow unlimited in the transverse direction.

\section{FRINGE EFFECTS IN SIMPLE ACCELERATING REGION}

The detailed exposition of the effects occurring in a drift region had as its main purpose to show that qualitative concepts about the formation of beam structure in the drift region can be transferred to the accelerating region. In any case, this is so when it is possible to rapidly control emission current, i.e., for the case of photocathodes.

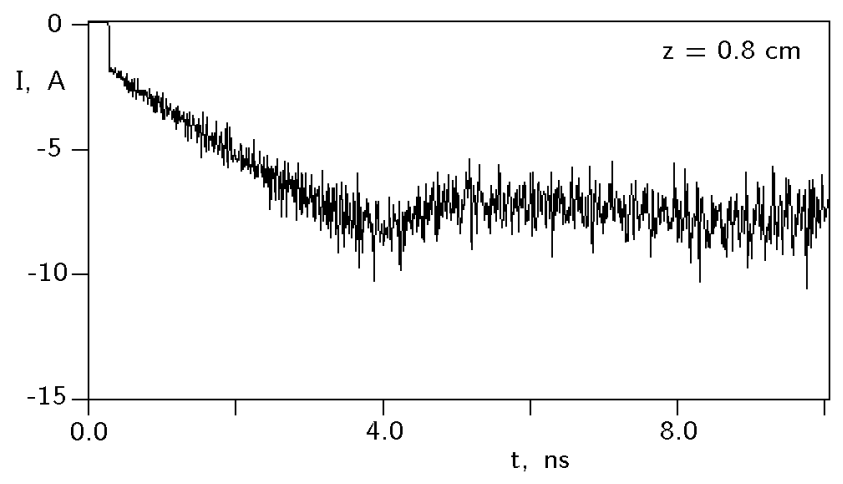

Figure. 4. Transient regime in plane diode

The formation of "whiskers" in a drift region occurs particularly effectively when close to the cathode boundary there is a conducting wall and particles simply drift along it with an energy close to the energy of injection. In the case of an accelerating region, the energy of emitted electrons is low and the formation of fringe "whiskers" is made difficult because of the low energy of "injection" and strong influence of the proper field. The proximity of the electrode, at cathode potential, directly to the boundary of the emitting surface leads to screening of the proper as well as extracting field. Thus, it is necessary that the distance of side walls from emitter edges be such as to allow, on the one hand, considerable screening the influence of own space charge and, on the other, penetration of a sufficiently large external field.

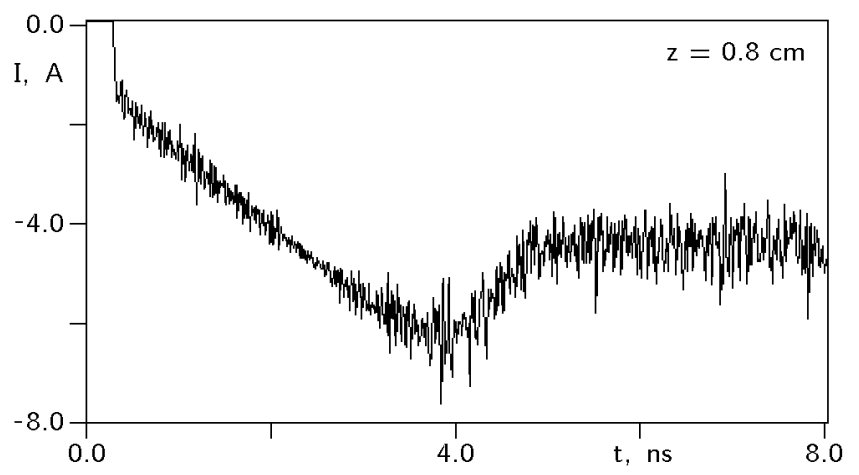

Figure. 5. Transient regime in conical diode

As an example that supports such an approach, we present in Fig. 4 and Fig. 5 time dependence of beam current in a plane diode and in a diode with emitting surface in a recess. The voltage on the diode is constant and equal to $20 \mathrm{kV}$, emitter diameter is $1 \mathrm{~cm}$, emission current increases linearly to $8 \mathrm{~A}$ in $4 \mathrm{~ns}$ and is then held constant. In the first case, hysteresis is practically imperceptible, but in the second it is quite pronounced. 


\section{HYSTERESIS EFFECTS IN A HIGH-VOLTAGE ELECTRON GUN}

Spatial-temporal hysteresis effects to a significant extent appeared in the process of developing an electron gun with large compression of a tubular beam (Fig. 6) intended for generation of powerful microwave and IR radiation [4].

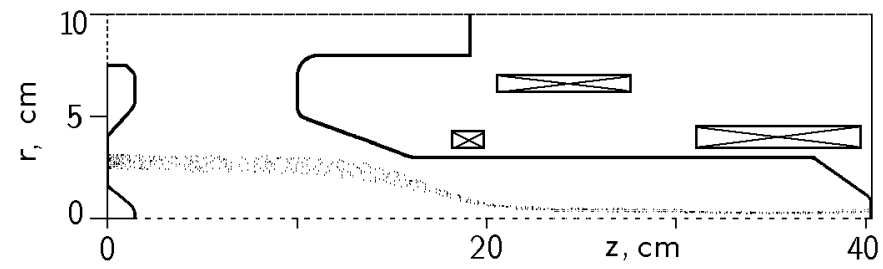

Figure. 6. Schematic diagram of the gun

Electromagnetic code KARAT was used to calculate beam dynamics and the results were tested by means of stationary code SAM [5]. Simulation of emission of particles in the KARAT code could be realized by two methods: giving constant (or variable) emission current exceeding the space-charge limited current and increasing the voltage on the gun to a certain given value (thermionic cathode); giving constant voltage on the gun and increasing emission current from the cathode in time in accordance with a desired law (photocathode).

Fig. 7 shows averaged (high-frequency components filtered off) behavior of beam current at the gun output for various modelling conditions. Dependencies 1-3 correspond to conditions of photocathode operation and dependence 4 to conditions of thermionic cathode operation. Dependencies 1-3 were obtained for constant rate of emission current increase (about $28 \mathrm{~A} / \mathrm{ns}$ ) on the linear front, the duration of which was $7 \mathrm{~ns}(195 \mathrm{~A}), 7.5 \mathrm{~ns}$ (210 A) and $11 \mathrm{~ns}(310 \mathrm{~A})$ for dependencies 1-3, respectively, and constant current for greater time. Current discrimination is clearly seen at $195 \mathrm{~A}$, with subsequent drop to values that approximately correspond to the value of beam current in the stationary state obtained with stationary code SAM. The fourth dependence is plotted for the case when emission current is held constant at $400 \mathrm{~A}$ and the voltage on the diode increases from $160 \mathrm{kV}$ to 400 $\mathrm{kV}$ in $8 \mathrm{~ns}$.

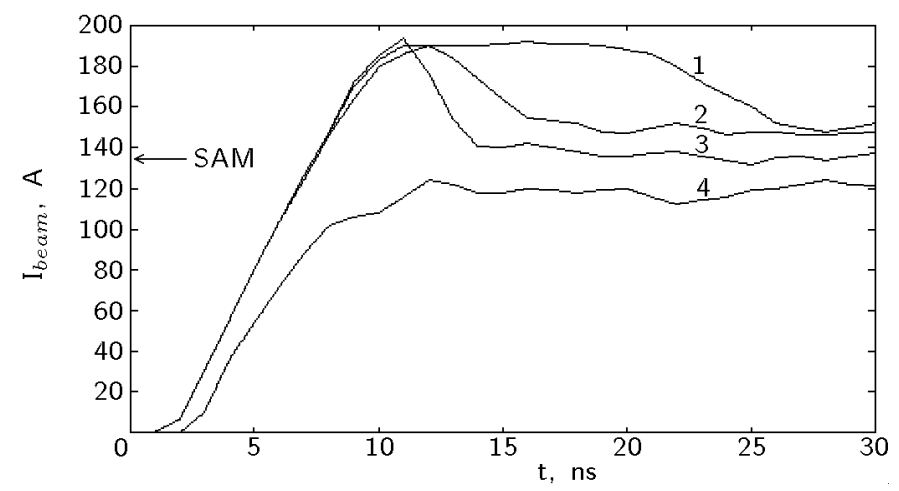

Figure. 7. Hysteresis effects in high-voltage gun

It should be noted that a little screening of the cathode (for example, placing it in a 5-mm deep circular slot), leading of course to a decrease in diode perveance, is accompanied by a sharper change in the ratio of peak to stationary value of current.

\section{REFERENCES}

[1] P. V. Kotetashwily, P. V. Rybak, V.P. Tarakanov, "KARAT - a Means for a Computer Experiment in Electrodynamics", Institute of General Physics, Moscow, Russia, Preprint no. 44, 1991.

[2] H. F. Ivey. Adv. Electronic and Electron Physics, v.6 (1954) $137-256$.

[3] V. S. Voronin, A. N. Lebedev, Y. T.Zozula. "Selfconsistent states of relativistic electron flow in a drift region", Sov. JTP, v.42 (1972) 546-551.

[4] A. V. Agafonov, E. G. Krastelev, A. N. Lebedev, V.S. Voronin, A.M. Bishaev, V. A. Krasnopolsky, P.J.M. van der Slot. "An electron gun with large compression of a thin annular beam". - Proc. 10th Intern. Conf. on High Power Particle Beams. June 20-24, 1994, San Diego, USA. Vol. 2, p. 522-525.

[5] M. A. Tiunov, B. M. Fomel, V. P. Yakovlev, "SAM - an Interactive Program for the Design of Electron Guns on a Minicomputers", Institute of Nuclear Physics, Novosibirsk, Russia, Preprint no. 87-35, 1987. 